\title{
0588. Effects of norepinephrine on tissue perfusion in a sheep model of intraabdominal hypertension
}

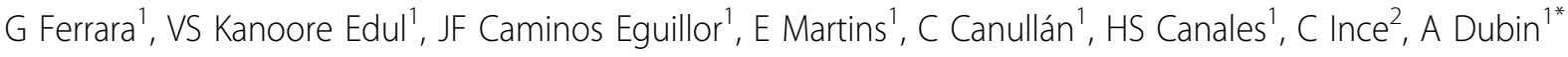 \\ From ESICM LIVES 2014 \\ Barcelona, Spain. 27 September - 1 October 2014
}

\section{Introduction}

Intraabdominal hypertension (IAH) produces detrimental effects on tissue perfusion. A putative underlying mechanism is the decrease in abdominal perfusion pressure (APP $=$ mean arterial pressure-intraabdominal pressure). Nevertheless, the benefits of increasing blood pressure on tissue perfusion are controversial.

\section{Objectives}

To describe the effects of IAH on regional and microcirculatory intestinal blood flow, renal blood flow, and urine output, as well as their responses to increases in blood pressure induced by norepinephrine.

\section{Methods}

In 24 anesthetized, mechanically ventilated, and fluidresuscitated sheep, we measured systemic hemodynamics, left renal and superior mesenteric artery blood flows, villi microcirculation, ileal intramucosal-arterial $\mathrm{PCO}_{2}\left(\Delta \mathrm{PCO}_{2}\right)$, and urine output. IAH $(20 \mathrm{~mm} \mathrm{Hg})$ was generated by intraperitoneal instillation of warmed saline. After $1 \mathrm{~h}$ of IAH, sheep were randomized to control $(\mathrm{n}=8)$ or norepinephrine $(\mathrm{n}=8)$ groups for $1 \mathrm{~h}$. In this last group, mean arterial pressure was increased about $20 \mathrm{~mm} \mathrm{Hg}$ by means of norepinephrine. A sham group $(\mathrm{n}=8)$ was also studied.

\section{Table 1}

\begin{tabular}{|c|c|c|c|c|c|c|c|c|}
\hline Period & Group & $\begin{array}{l}\text { APP } \\
(\mathrm{mm} \\
\mathrm{Hg})\end{array}$ & $\begin{array}{l}\text { Cardiac output } \\
\left(\mathrm{mL} \cdot \mathrm{min}^{-1} \cdot \mathrm{kg}^{-1}\right)\end{array}$ & $\begin{array}{l}\text { Mesenteric flow } \\
\left(\mathrm{mL} \cdot \mathrm{min}^{-1} \cdot \mathrm{kg}^{-1}\right)\end{array}$ & $\begin{array}{l}\triangle \mathrm{PCO}_{2} \\
(\mathrm{~mm} \mathrm{Hg})\end{array}$ & $\begin{array}{l}\text { Villi perfused density } \\
\left(\mathrm{mm} / \mathrm{mm}^{2}\right)\end{array}$ & $\begin{array}{l}\text { Renal flow (mL. } \\
\min ^{-1} \cdot \mathrm{kg}^{-1} \text { ) }\end{array}$ & $\begin{array}{l}\text { Urine output } \\
\left(\mathrm{mL} \cdot \mathrm{min} \cdot \mathrm{kg}^{-1}\right)\end{array}$ \\
\hline \multirow[t]{3}{*}{ Basal } & Control & $83 \pm 12$ & $122 \pm 26$ & $392 \pm 154$ & $7 \pm 6$ & $22 \pm 3$ & $1906 \pm 517$ & $1.2 \pm 0.3$ \\
\hline & Norepinephrine & $82 \pm 7$ & $96 \pm 17$ & $445 \pm 318$ & $11 \pm 7$ & $26 \pm 3$ & $1905 \pm 729$ & $1.1 \pm 0.5$ \\
\hline & Sham & $76 \pm 13$ & $113 \pm 18$ & $405 \pm 115$ & $4 \pm 4$ & $23 \pm 4$ & $1890 \pm 639$ & $1.4 \pm 0.6$ \\
\hline \multirow[t]{3}{*}{$\begin{array}{l}1-\mathrm{h} \\
\mathrm{IAH}\end{array}$} & Control & $55 \pm 10$ & $121 \pm 41$ & $524 \pm 302$ & $6 \pm 6$ & $25 \pm 3$ & $943 \pm 416$ & $0.4 \pm 0.1$ \\
\hline & Norepinephrine & $53 \pm 9$ & $118 \pm 42$ & $633 \pm 383$ & $12 \pm 5$ & $27 \pm 3$ & $552 \pm 359$ & $0.4 \pm 0.7$ \\
\hline & Sham & $87 \pm 14^{*}$ & $120 \pm 28$ & $449 \pm 88$ & $4 \pm 6$ & $24 \pm 3$ & $1730 \pm 510^{*}$ & $0.9 \pm 0.5^{*}$ \\
\hline \multirow[t]{3}{*}{$\begin{array}{l}2-h \\
\mathrm{IAH}\end{array}$} & Control & $49 \pm 18^{*}$ & $134 \pm 39$ & $522 \pm 322$ & $8 \pm 6$ & $24 \pm 2$ & $869 \pm 612$ & $0.3 \pm 0.4$ \\
\hline & Norepinephrine & $73 \pm 10$ & $113 \pm 39$ & $634 \pm 310$ & $12 \pm 6$ & $28 \pm 3$ & $620 \pm 439$ & $0.2 \pm 0.1$ \\
\hline & Sham & $87 \pm 15$ & $127 \pm 24$ & $448 \pm 108$ & $3 \pm 5$ & $25 \pm 4$ & $1678 \pm 569^{*}$ & $1.0 \pm 0.6^{*}$ \\
\hline
\end{tabular}

${ }^{*} p<0.05$ vs. the other groups ( $t$ test with Bonferroni correction after significant time $x$ group interaction in two-way repeated measures of ANOVA).

\footnotetext{
${ }^{1}$ Facultad de Ciencias Médicas, Universidad Nacional de La Plata, Cátedra de

Farmacología Aplicada, La Plata, Argentina

Full list of author information is available at the end of the article
} 


\section{Conclusions}

In this experimental model of IAH, the gut and the kidney displayed contrasting responses. While intestinal blood flow and villi microcirculation remained unchanged, renal perfusion and urine output were severely compromised. The increase in blood pressure with norepinephrine failed to improve these variables.

\section{Grant acknowledgment}

This work was supported by the grant PICT-2010-0495 from Agencia

Nacional de Promoción Científica y Tecnológica, Argentina.

\section{Authors' details}

${ }^{1}$ Facultad de Ciencias Médicas, Universidad Nacional de La Plata, Cátedra de Farmacología Aplicada, La Plata, Argentina. ${ }^{2}$ Academic Medical Center,

Department of Translational Physiology, Amsterdam, Netherlands.

Published: 26 September 2014

doi:10.1186/2197-425X-2-S1-P33

Cite this article as: Ferrara et al:: 0588. Effects of norepinephrine on tissue perfusion in a sheep model of intraabdominal hypertension. Intensive Care Medicine Experimental 2014 2(Suppl 1):P33.

\section{Submit your manuscript to a SpringerOpen ${ }^{\circ}$ journal and benefit from:}

- Convenient online submission

- Rigorous peer review

- Immediate publication on acceptance

- Open access: articles freely available online

- High visibility within the field

- Retaining the copyright to your article 